\title{
A novel method for synthesizing PEGylated chitosan nanoparticles: strategy, preparation, and in vitro analysis
}

This article was published in the following Dove Press journal:

International Journal of Nanomedicine

2 March 2011

Number of times this article has been viewed

\section{Meenakshi Malhotra \\ Ciaran Lane \\ Catherine \\ Tomaro-Duchesneau \\ Shyamali Saha \\ Satya Prakash}

Biomedical Technology and Cell

Therapy Research Laboratory,

Departments of Biomedical

Engineering and Physiology, Artificial

Cells and Organs Research Center,

Faculty of Medicine, McGill University,

Quebec, Canada
Correspondence: Satya Prakash

3775 University Street, Montreal,

Quebec, H3A 2B4, Canada

$\mathrm{Tel}+$ I 5 I4 3982736

Fax +| $5|4398746|$

Email satya.prakash@mcgill.ca
Abstract: Preparation of poly (ethylene glycol) (PEG)-grafted chitosan is essential for improving the biocompatibility and water solubility of chitosan. Presently available methods for this have limitations. This article describes a new method for preparing PEGylated chitosan nanoparticles. For this chitosan was chemoselectively modified using a novel scheme at the $\mathrm{C} 6$ position of its repeating units by PEG. The amine groups at the $\mathrm{C} 2$ position of the chitosan were protected using phthalic anhydride. Sodium hydride was used to catalyze the etherification reaction between chlorinated chitosan and methyl-PEG, and PEG-grafted chitosan was successfully synthesized. Each step was characterized using ${ }^{13} \mathrm{C}$ nuclear magnetic resonance and Fourier transform infrared. After PEGylation the phthaloylated chitosan was successfully deprotected using hydrazine monohydrate. The synthetic scheme proposed demonstrates a new method for grafting PEG onto chitosan with a moderate degree of substitution. The potential of this polymer in nanoparticle preparation using an ionic gelation method and its gene delivery potentials were investigated by complexing a fluorescently labeled control siRNA. The result showed that suitable nanoparticles can be synthesized using this polymer and that they have capacity to carry genes and provide adequate transfection efficacy with no toxicity when tested in neuronal cells.

Keywords: nanoparticles, chitosan, poly (ethylene glycol), polymeric membrane, gene delivery

\section{Introduction}

Chitosan is a linear polymer composed of $\beta$-(1-4)-2-amino-2-deoxy-D-glucopyranose units. Being a polycationic, nontoxic, biodegradable, and biocompatible polymer, chitosan has attracted much attention and has wide applications in biotechnology, pharmaceutical, textile, food, cosmetics, and agricultural industries. ${ }^{1,2}$ Current research with chitosan focuses on its use as a novel drug, gene, peptide, and vaccine delivery vehicle, and scaffold for targeted delivery and tissue engineering applications. ${ }^{3-6}$ Chitosan has achieved much interest compared with chitin and cellulose due to the presence of primary amine groups present in its repeating units. The presence of primary amine groups makes chitosan an excellent cell transfectant. Like polyethyleneimine, chitosan exhibits a "proton sponge" effect, which refers to the swelling behavior of the polymer on encountering an acidic $\mathrm{pH}$ inside the cell's endosome, making it an efficient carrier for therapeutic molecules..$^{7-9}$ A number of chitosan derivatives have been synthesized in the past with modification on the primary amine groups of the polymer. ${ }^{10,11}$ Chitosan is soluble only in acidic aqueous solutions due to the presence of amine groups that become protonated, and thus its poor solubility in other organic solvents has become a major drawback, limiting its effective utilization. 
To facilitate the effective use of chitosan in gene delivery and other biomedical applications, it is necessary to make chitosan soluble either in water or organic solvents. Such attempts can be made by graft copolymerization of chitosan through chemical modifications with other polymers, such as poly (ethylene glycol) (PEG), of different molecular weights. ${ }^{12}$ This allows chitosan to retain its inherent characteristics of, for example, molecular structure and length, and allows for a variety of chemical modifications or reactions on its side chain. However, chemical modification at amine groups leads to a change in the fundamental skeleton of chitosan, causing it to lose its original physiochemical and biochemical activities. ${ }^{12}$ Recently, modifying hydroxyl groups of chitosan has gained much importance as it does not influence the characteristic structural and functional features of chitosan.

PEG is widely used as a graft-forming polymer. It is soluble in both water and organic solvents, has no toxicity, antigenicity, and immunogenicity, and is biodegradable and biocompatible. ${ }^{13}$ PEGylation of chitosan through hydroxyl groups was first proposed by Gorochovceva and Makuska. ${ }^{14}$ PEG has been used mainly as a graft polymer, where it is used as a crosslinker and forms interconnected channels to enable drug release. ${ }^{15,16}$ Various preparation techniques can be used to prepare chitosan nanocarriers by altering parameters such as concentration of the polymer or the crosslinker, the molecular weight of chitosan, the ratio of drug/gene:polymer, $\mathrm{pH}$, and finally stabilizers or surfactants. All these factors collectively affect the structural and morphological properties of chitosan nanoparticles and the release rate of the loaded therapeutic molecule. ${ }^{17}$

This study aims to achieve facile chemoselective conjugation of PEG at the hydroxyl group of chitosan, where the amine groups of chitosan were first protected using phthalic anhydride. ${ }^{18}$ Nanoparticles were formed using an ionic gelation method. The concentration of the polymer versus crosslinker and the $\mathrm{pH}$ of the solution were adjusted with reference to our previously optimized study. ${ }^{19}$ The current study opens up the future application of forming PEG-grafted chitosan nanoparticles for efficient gene/drug delivery.

\section{Materials and methods Materials}

Low-molecular-weight chitosan was obtained from Wako (Richmond, VA, USA), having a viscosity of 5-20 cP and a degree of deacetylation of $80.0 \%$. Poly (ethylene glycol) monomethyl ether (MW 5000), phthalic anhydride, sodium hydride $(\mathrm{NaH})$, pyridine, hydrazine monohydrate, sodium tripolyphosphate (TPP), glacial acetic acid, agarose (low gelling temperature), and ethidium bromide $(10 \mathrm{mg} / \mathrm{mL})$ of analytical grade were obtained from Sigma-Aldrich (Oakville, ON, Canada). Anhydrous tetrahydrofuran (THF), anhydrous N,N-dimethylformamide (DMF), sodium hydroxide $(\mathrm{NaOH})$ and methanol were obtained from Thermo Fisher Scientific (Ottawa, ON, Canada). Thionyl chloride $\left(\mathrm{SOCl}_{2}\right)$ was obtained from VWR (Mississauga, ON, Canada). For dilution purposes, ultra pure doubledistilled water $\left(\mathrm{ddH}_{2} \mathrm{O}\right)$ was used from a laboratory-installed Barnstead Nanopure diamond ${ }^{\mathrm{TM}}$ water supply unit. siGLO (Green) transfection indicator (20 nmole) was obtained from Dharmacon (Lafayette, IN, USA). CellTiter $96^{\circledR}$ AQueous One Solution Reagent was purchased from Promega (Madison, WI, USA) to perform cell viability assay. TrackIt 10 bp DNA ladder $(0.5 \mu \mathrm{g} / \mu \mathrm{L})$ was obtained from Invitrogen (Burlington, ON, Canada). Neuro2a cell line and Eagle's minimum essential medium (EMEM) were obtained from Cedarlane (Burlington, ON, Canada) and supplemented with $10 \%$ fetal bovine serum (FBS) from Invitrogen (Burlington, ON, Canada).

\section{Deacetylation of chitosan}

Commercially available low-molecular-weight chitosan (5g) was added to a $40 \%$ (w/v) aqueous $\mathrm{NaOH}$ solution. The mixture was stirred for 4 hours at $110^{\circ} \mathrm{C}$ under a nitrogen atmosphere. After 4 hours the mixture was vacuum filtered,

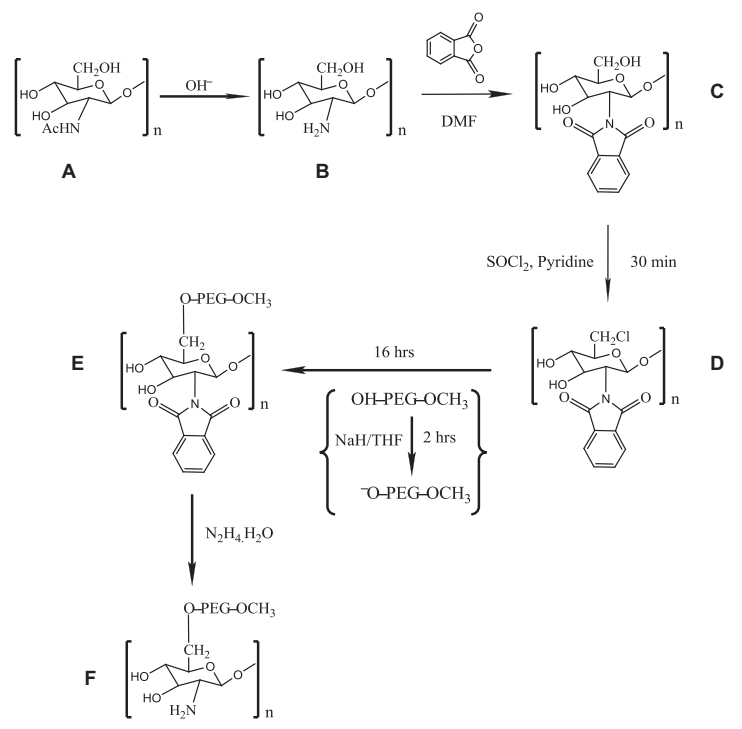

Figure I Schematic of O-PEGylated chitosan polymer preparation using $\mathrm{NaH}$ and THF: A) chitosan; B) deacetylated chitosan; C) phthaloyl chitosan; D) chlorinated phthaloyl chitosan intermediate; E) PEGylated phthaloyl chitosan; and F) PEGylated chitosan.

Abbreviations: $\mathrm{NaH}$, sodium hydride; THF, tetrahydrofuran. 
pulverized, and was again treated with $40 \%$ (w/v) $\mathrm{NaOH}$ under similar conditions. The resultant product (b) in Figure 1 was freeze-dried. ${ }^{20}$

\section{Phthaloylation of chitosan}

Deacetylated chitosan (1.00 g) was added to a solution of phthalic anhydride $(2.76 \mathrm{~g})$ in $20 \mathrm{~mL}$ of DMF. The mixture was stirred for 8 hours at $120^{\circ} \mathrm{C}$ under a nitrogen atmosphere. The resultant product (c) in Figure 1 was cooled to room temperature and precipitated in ice cold water. The precipitate was filtered, washed with methanol overnight, and vacuum dried. ${ }^{18,21}$

\section{Synthesis of PEGylated chitosan (chitosan-O-PEG)}

We employed a novel scheme to PEGylate chitosan chemoselectively. The scheme depicted in Figure 1 utilizes the activation of PEG by $\mathrm{NaH}$, used as a catalyst to conjugate PEG and chitosan. The synthesis was accomplished in 3 steps: 1) chlorination of chitosan, 2) activation of OH-PEG-OCH (mPEG) with $\mathrm{NaH}$, and 3) grafting activated mPEG onto chlorinated chitosan. To prepare chlorinated chitosan, $\mathrm{SOCl}_{2}$ was added in 10-fold excess compared to phthaloylated chitosan $(0.1 \mathrm{~g})$ in $20 \mathrm{~mL}$ of pyridine. The reaction was stirred at $80^{\circ} \mathrm{C}$ for 30 minutes under a nitrogen atmosphere. After 30 minutes, the reaction was cooled to room temperature, precipitated in ice cold water, filtered, and vacuum dried to yield chlorinated-phthaloyl chitosan, product (d) in Figure 1. PEG was activated by adding $4 \mathrm{~g}$ of OH-PEG-OCH (mPEG) $_{3}$ to a suspension of $\mathrm{NaH}(10 \mathrm{mg})$ in $50 \mathrm{~mL}$ of anhydrous THF. The reaction was stirred at $60^{\circ} \mathrm{C}$ for 2 hours under nitrogen atmosphere. After 2 hours, chlorinated-phthaloyl chitosan $(60 \mathrm{mg}$ ) was added to the reaction mix and stirred for another 16 hours under similar conditions. After 16 hours the reaction was allowed to cool at room temperature and was precipitated in methanol, filtered, and vacuum dried to yield product (e) in Figure 1.

\section{Deprotection of PEGylated-phthaloyl chitosan}

PEGylated-phthaloyl chitosan (100 mg), hydrazine monohydrate $(15 \mathrm{~mL})$, and distilled water $(30 \mathrm{~mL})$ were mixed and heated at $100^{\circ} \mathrm{C}$ for 16 hours under constant magnetic stirring. Excess hydrazine monohydrate was removed by evaporating the mixture using a rotary evaporator until a viscous solution was left. The process was repeated 3 times by reconstituting the mix with distilled water each time and then rotary evaporating it until a solid residue was left. The final product was dried under vacuum to obtain the desired PEGylated chitosan.

\section{Preparation of PEGylated chitosan nanoparticles}

PEGylated chitosan nanoparticles were prepared by an ionic gelation procedure, as explained in previous studies. ${ }^{22}$ After the deprotection of PEGylated-phthaloyl chitosan, the polymer was dissolved in $1 \%(\mathrm{v} / \mathrm{v})$ acetic acid solution to yield a concentration of $0.5 \mathrm{mg} / \mathrm{mL}$ and the $\mathrm{pH}$ was adjusted to 5 . TPP was dissolved in $\mathrm{ddH}_{2} \mathrm{O}$ to obtain a concentration of $0.7 \mathrm{mg} / \mathrm{mL}$ and the $\mathrm{pH}$ was adjusted to $3 .{ }^{19}$ Nanoparticles were formed after the addition of TPP (drop-wise) to the chitosan solution under constant magnetic stirring for 1 hour at room temperature. The nanoparticles obtained were characterized by TEM for their morphology and size.

\section{Gene loading efficiency by the gel retardation assay}

The encapsulation/gene loading efficiency of PEGylated chitosan polymer was determined by complexing it with siGLO, a transfection indicator, to form nanoparticles following similar conditions as described in section 2.6. The free siGLO in PBS and siGLO complexed with nanoparticles were run in duplicates on a $4 \% \mathrm{w} / \mathrm{v}$ agarose gel electrophoresis for 4 hours at $55 \mathrm{~V}$ in Tris/Borate/EDTA (TBE) buffer ( $\mathrm{pH} 8.3$ ). The siGLO complexation with nanoparticles was performed at a ratio of 200:1 (polymer:gene) (w/w), as determined in our previous study. ${ }^{19}$ The TBE buffer used contained ethidium bromide at a concentration of $0.5 \mu \mathrm{g} / \mathrm{mL}$, which is required for the visualization of the RNA bands under UV transilluminator at a wavelength of $365 \mathrm{~nm}$ using a ChemiDoc ${ }^{\mathrm{TM}}$ XRS System (Bio-Rad, Hercules, CA).

\section{Transfection efficiency and cell viability assay}

Mouse neuroblastoma cells (Neuro2a) were seeded in a 96-well plate at a density of 20,000 cells per well in complete growth media (antibiotics-free EMEM with $10 \%$ serum). The transfection study was performed after 24 hours of initial seeding. The different tested samples were: chitosan-TPP nanoparticles and free siGLO as controls, chitosan-TPP:siGLO nanoparticles, and PEGylated chitosan-TPP:siGLO nanoparticles (200:1 w/w) as treatment samples. The cells were then incubated at $37^{\circ} \mathrm{C}$, $5 \% \mathrm{CO}_{2}$ for 4 hours, after which the cells were observed under fluorescence microscope (Nikon Eclipse TE2000-U) 
at a wavelength of $490 \mathrm{~nm}$. The same treatment and control samples were also used for a cytotoxicity assay. The cytotoxicity assay was performed after 4 hours using CellTiter $96^{\circledR}$ AQueous One Solution Reagent (MTS assay), as per manufacturer's protocol. After an additional incubation of 4 hours with the MTS assay the absorbance in each well was recorded at $490 \mathrm{~nm}$ using a microtiter plate reader (Perkin Elmer $^{\circledR} 1420$ Multilabel Counter Victor 3TM V). The cell study was performed in triplicate.

\section{Statistical analysis}

Values are expressed as means \pm standard deviations (SD). The statistical significance was determined by using an independent $t$-test to compare the values of chitosan-TPP nanoparticles with PEGylated chitosan-TPP nanoparticles ( $n=3$ for each group). $P$ values of $<0.05$ were considered significant. Statistical analysis was carried out using Minitab (Minitab, Version 14; Minitab Inc, State College, PA).

\section{Results \\ Deacetylation of chitosan}

The ${ }^{13} \mathrm{C}$ nuclear magnetic resonance (NMR) of deacetylated chitosan confirmed the absence of an acetyl peak $(-\mathrm{CH} 3)$ at $23.88 \mathrm{ppm}$ and a carbonyl peak $(-\mathrm{C}=\mathrm{O})$ at $175.04 \mathrm{ppm}$ present in the commercially available chitosan. NMR: (topological substructural molecular design (TOSS mode) of commercially available chitosan: $\delta_{\mathrm{C}} 23.88\left(\mathrm{CH}_{3}\right), 59.01$ (C-2), 61.76 (C-6), 75.64 (C-5, 3), 82.51 (C-4), 105.68 (C-1), $175.04(\mathrm{C}=\mathrm{O}) .{ }^{13} \mathrm{C}$ CP/MAS NMR: (TOSS mode) of deacetylated chitosan: $\delta_{\mathrm{C}} 60.76(\mathrm{C}-2,6), 75.84(\mathrm{C}-4,5,3)$, 102.80 (C-1).

\section{Phthaloylation of chitosan}

The ${ }^{13} \mathrm{C}$ NMR in TOSS mode of phthaloylated chitosan confirmed the appearance of peaks Phth phenylene and Phth $\mathrm{C}=\mathrm{O}$ at $134.42 \mathrm{ppm}$ and $169.66 \mathrm{ppm}$, respectively, and the appearance of Phth $\mathrm{C}-1,2$ and $\mathrm{Phth} \mathrm{C}=\mathrm{O}$ peaks at 131.63 ppm and 169.89 ppm, respectively, in TOSDL mode. ${ }^{13} \mathrm{C}$ CP/MAS NMR: (TOSS mode): $\delta_{\mathrm{C}} 58.19$ (C-2), 61.76 (C-6), 72.44 (C-3), 75.42 (C-5), 83.91 (C-4), 101.24 (C-1), 124.62, 131.70, 134.42 (Phth phenylene), and 169.66 (Phth $\mathrm{C}=\mathrm{O})$; (TOSDL mode): $\delta_{\mathrm{C}} 131.63$ (Phth $\left.\mathrm{C}-1,2\right)$ and 169.89 (Phth $\mathrm{C}=\mathrm{O}$ ). Fourier transform infrared (FTIR) of phthaloylated chitosan as represented in Figure $2 \mathrm{~b}: \mathrm{v}_{\max } / \mathrm{cm}^{-1}$ 3200-3400 (OH), 1774 (imide $\mathrm{C}=\mathrm{O}$ ), 1710 (imide $\mathrm{C}=\mathrm{O}$ ), 1150-1000 (pyranose), and 720 (arom). FTIR of commercially available chitosan as shown in Figure $2 \mathrm{a}: \mathrm{v}_{\max } / \mathrm{cm}^{-1} 1630$ (amide I), 1542 (amide II), and 1024 (pyranose).

\section{Synthesis of PEGylated chitosan}

The FTIR spectra presented in Figure $2 \mathrm{~d}$ represent PEGylated chitosan with the characteristic peaks at $v_{\max } / \mathrm{cm}^{-1}: 2871(\mathrm{C}-\mathrm{H}$ stretching), 1066 (C-O stretching), 1290, 1251, 950 and 837, confirming the $\mathrm{PEG}_{5000}$ substitution when compared to Figure $2 \mathrm{c}$ that represents the FTIR spectra of PEG only with the characteristic peaks at $\mathrm{v}_{\max } / \mathrm{cm}^{-1}: 2878$ (C-H stretching), 1100 (C-O stretching), 1466, and 1278.

\section{Deprotection of PEGylated-phthaloyl chitosan}

The FTIR spectra presented in Figure 2e represent deprotected PEGylated chitosan with the characteristic peaks at $v_{\max } / \mathrm{cm}^{-1}: 2878$ (C-H stretching), 1066 (C-O stretching) belonging to PEG, the appearance of 1633 (amide I), 1582 (amide II), and 1024 (pyranose) belonging to chitosan, and the disappearance of 1774 (imide $\mathrm{C}=\mathrm{O}$ ), 1710 (imide $\mathrm{C}=\mathrm{O}$ ) belonging to the phthaloyl group, as shown in Figure $2 \mathrm{~b}$.

\section{Preparation of PEGylated chitosan nanoparticles}

Deprotected PEGylated chitosan polymer was crosslinked with TPP by the electrostatic interaction between the cationic charges of the primary amine groups on chitosan with the anionic charges of TPP. The crosslinking results in the formation of nanoparticles ranging from 100 to $150 \mathrm{~nm}$ in size as determined by transmission electron microscopy (TEM). Figure 3 a represents the TEM image of the PEGylated chitosan polymer (magnification: $57000 \mathrm{X})$. Figure $3 \mathrm{~b}$ represents the chitosan-TPP nanoparticles (magnification: 22000X) and Figure 3c and $3 \mathrm{~d}$ represents the PEGylated chitosan-TPP nanoparticles at magnification $57000 \mathrm{X}$ and $135000 \mathrm{X}$ respectively. It was observed that PEGylation yielded a spherical shape to the nanoparticles.

\section{Gene loading efficiency of PEGylated chitosan nanoparticles}

In order to evaluate the complexation of siGLO with PEGylated chitosan-TPP nanoparticles, a gel retardation assay was performed, wherein a $10 \mathrm{bp}$ DNA ladder was used as a reference, free siGLO at a concentration of $6 \mu \mathrm{g} / \mathrm{mL}$ of PBS solution was used as the control and PEGylated chitosan-TPP nanoparticles complexing siGLO at a ratio of 200:1 (w/w), as determined in our previous study, 


\section{E}

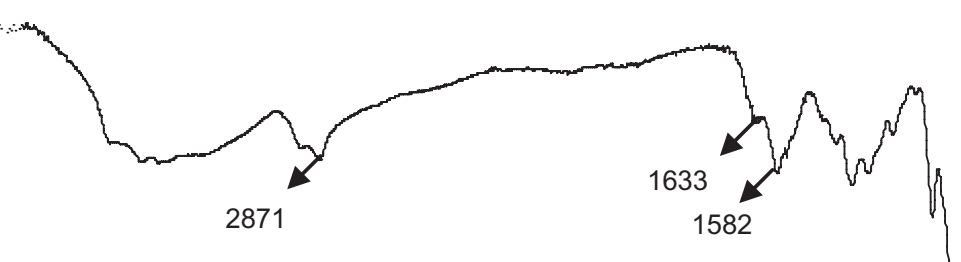

D

C

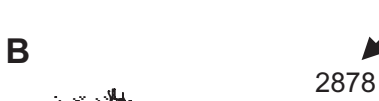

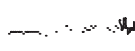
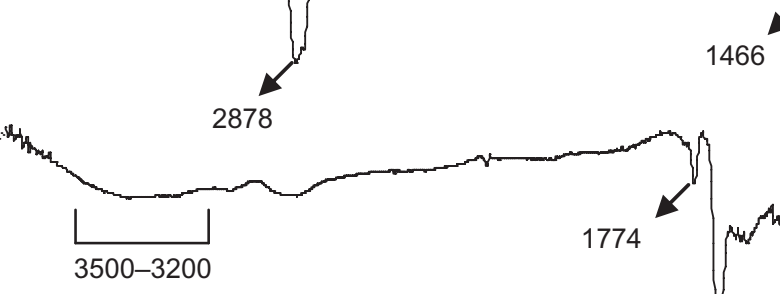

A

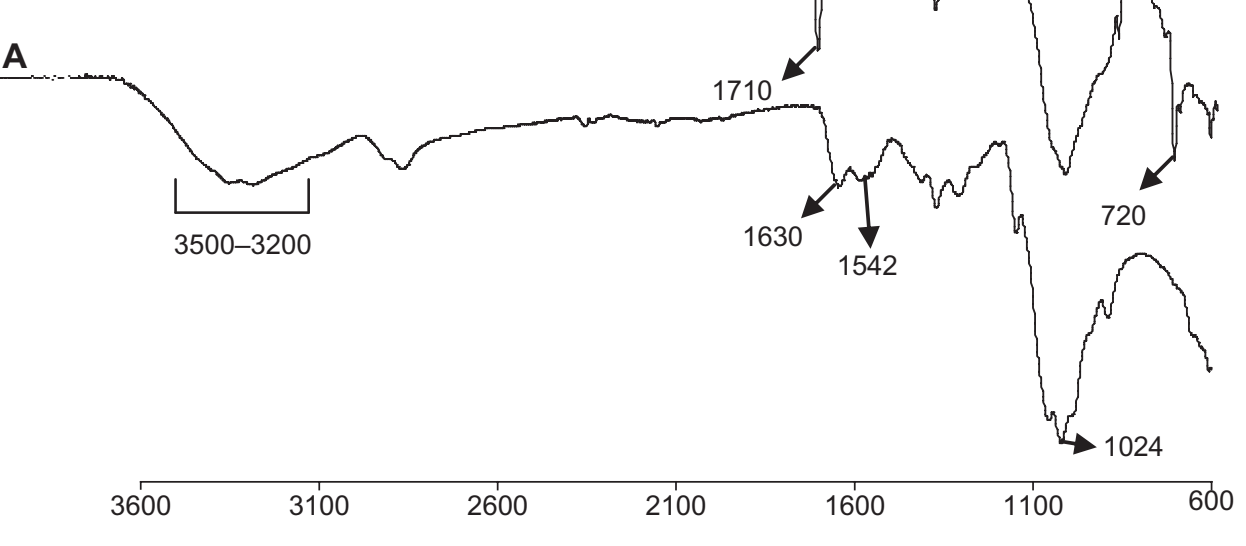

Figure 2 Fourier transform infrared spectra of the chitosan intermediates and O-PEGylated chitosan: A) deacetylated chitosan; B) phthaloylated chitosan: peaks at $1774 \mathrm{~cm}^{-1}$ and $1702 \mathrm{~cm}^{-1}$. The $\mathrm{OH}$ groups of phthaloylated chitosan was chlorinated using thionyl chloride, represented by the reduction in peak: from 3500 to $3200 \mathrm{~cm}^{-1}$; C) $\mathrm{OH}-\mathrm{PEG}-\mathrm{OCH}_{3}$, M.W. 5,000; D) 6-O-PEG-g-2-N-phthaloyl chitosan; E) PEG-grafted chitosan: disappearance of peaks $1774 \mathrm{~cm}^{-1}$ and $I 702 \mathrm{~cm}^{-1}$.

was used as the treatment sample. ${ }^{19}$ The gel, under a UV transilluminator, shows bands indicating the presence of unbound siGLO in the suspension, as shown in Figure 4, for the control sample (ie, free siGLO in PBS), whereas there is no band in a treatment sample, which confirms the complete complexation of the siGLO with PEGylated chitosan nanoparticles. The strong interaction pertains to the positive charges available on the chitosan chain after the deprotection procedure on the polymer allowing the interaction with the negatively charged siGLO and TPP. 

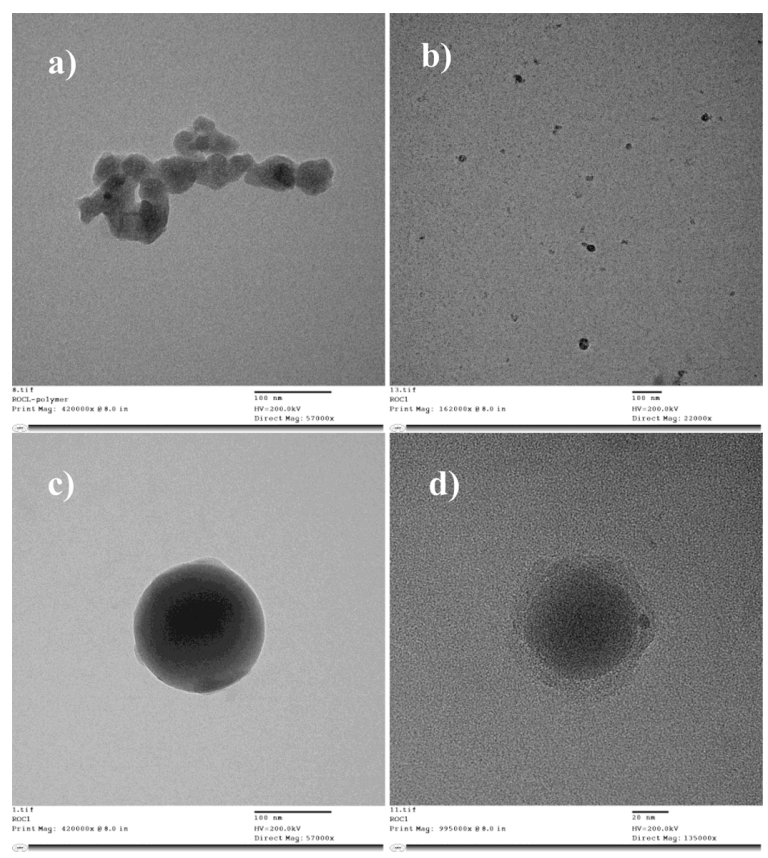

Figure 3 TEM images of a) PEGylated chitosan polymer (mag. 57,000x); b) chitosanTPP nanoparticle (mag. 22,000x); c) PEGylated chitosan-TPP nanoparticle (mag. 57,000x) and d), PEGy lated chitosan-TPP nanoparticle (mag. 135000x).

Abbreviation: TPP, sodium tripolyphosphate.

\section{Transfection efficiency and cell viability assay}

The transfection efficiency was analyzed by measuring the fluorescence intensity of the siGLO-loaded nanoparticles. siGLO is a transfection indicator, which is a fluorescent oligonucleotide that is localized in the nucleus of the mammalian cell (Figure 5). Free siGLO, chitosan-TPP nanoparticles, and cells without any treatment sample were used as negative controls. An efficient transfection efficiency was observed with both chitosan-TPP:siGLO and PEGylated chitosan-TPP:siGLO nanoparticles prepared at a weight ratio of 200:1 (Figure 5). The cell viability assay was performed on these cells after 4 hours, wherein it was observed that cells treated with PEGylated chitosanTPP:siGLO nanoparticles showed significant increase in cell viability $(\approx 70 \%)$ compared with the chitosan-TPP:siGLO nanoparticles $(\approx 20 \%)$ (independent $t$-test, $P<0.05$ ), as observed in Figure 6.

\section{Discussion}

The degree of $\mathrm{N}$-acetylation of chitin differentiates it from chitosan. The lower degree of $\mathrm{N}$-acetylation of chitosan widens its application for chemical modifications with the amine groups, imparts greater solubility to the polymer, and enhances its biological properties. Unlike chitin, chitosan is

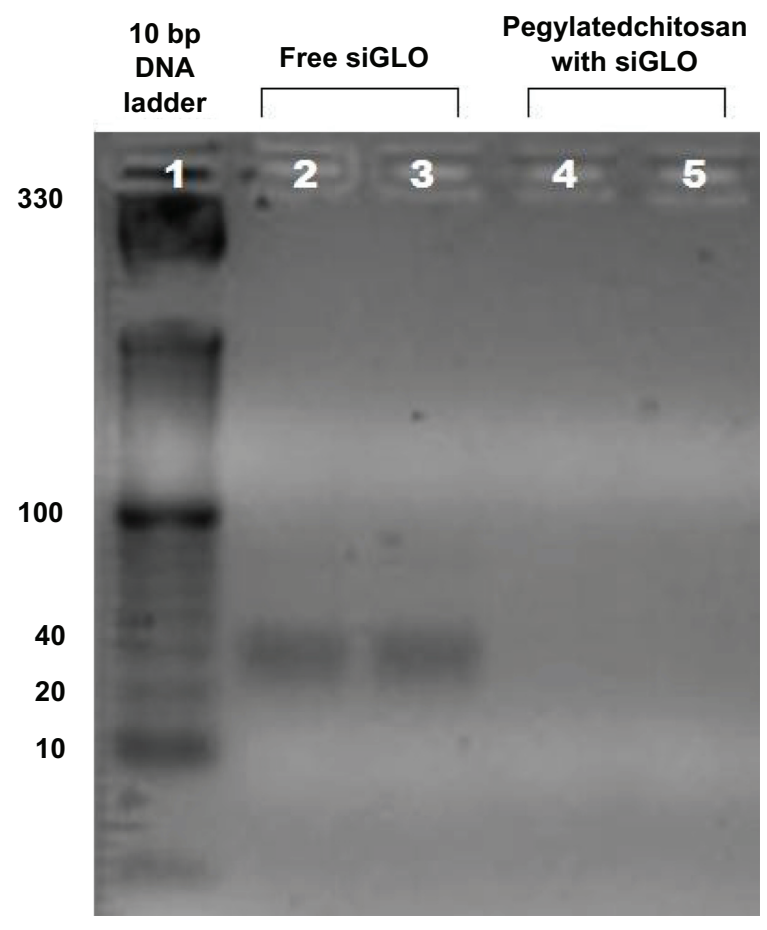

Figure $4 \mathrm{Gel}$ retardation assay: lane I) control 10 bp DNA ladder; lane 2 and 3 control siGLO, and lane 4 and 5 are PEGylated chitosan complexed siGLO at a ratio of 200:I (w/w). In all groups the siGLO concentration was kept constant at $6 \mu \mathrm{g} / \mathrm{mL}$. The disappearance of the band size at 40 bp in PEGylated chitosan complexed siGLO (lanes 4 and 5), compared to siGlO control (lanes 2 and 3), indicates the complete complexation of the siGLO.

soluble only in acidic aqueous solutions. The solubility of the polymer is an important parameter for having a homogeneous reaction mixture in order to obtain successful chemical modifications. The solubility of chitosan also depends on its molecular weight, its degree of neutralization of amine groups, the ionic strength of the solvent, and the distribution of $\mathrm{N}$-acetyl glucosamine residues along the backbone of the chain. ${ }^{23}$ In these experiments complete deacetylation of chitosan was achieved after treating it twice with a $40 \%(\mathrm{w} / \mathrm{v})$ $\mathrm{NaOH}$ solution. Products (a) and (b) in Figure 1 represent the schematic of acetylated chitosan (commercially available) and deacetylated chitosan, respectively.

The complete deacetylation is required to achieve a chitosan with fully activated amine groups allowing for further chemical reactions but renders crystalline character to the polymer, making it insoluble in most organic solvents. ${ }^{21}$ In order to solubilize chitosan in organic solvents and to avoid the interference of amine groups while performing chemical modifications on the backbone, the amine groups of chitosan were protected using phthalic anhydride. ${ }^{21}$ Since it has been observed that phthaloylation of chitosan in 100\% DMF yields partial O-phthaloylation along with N-phthaloylation, we attempted to use DMF/water 

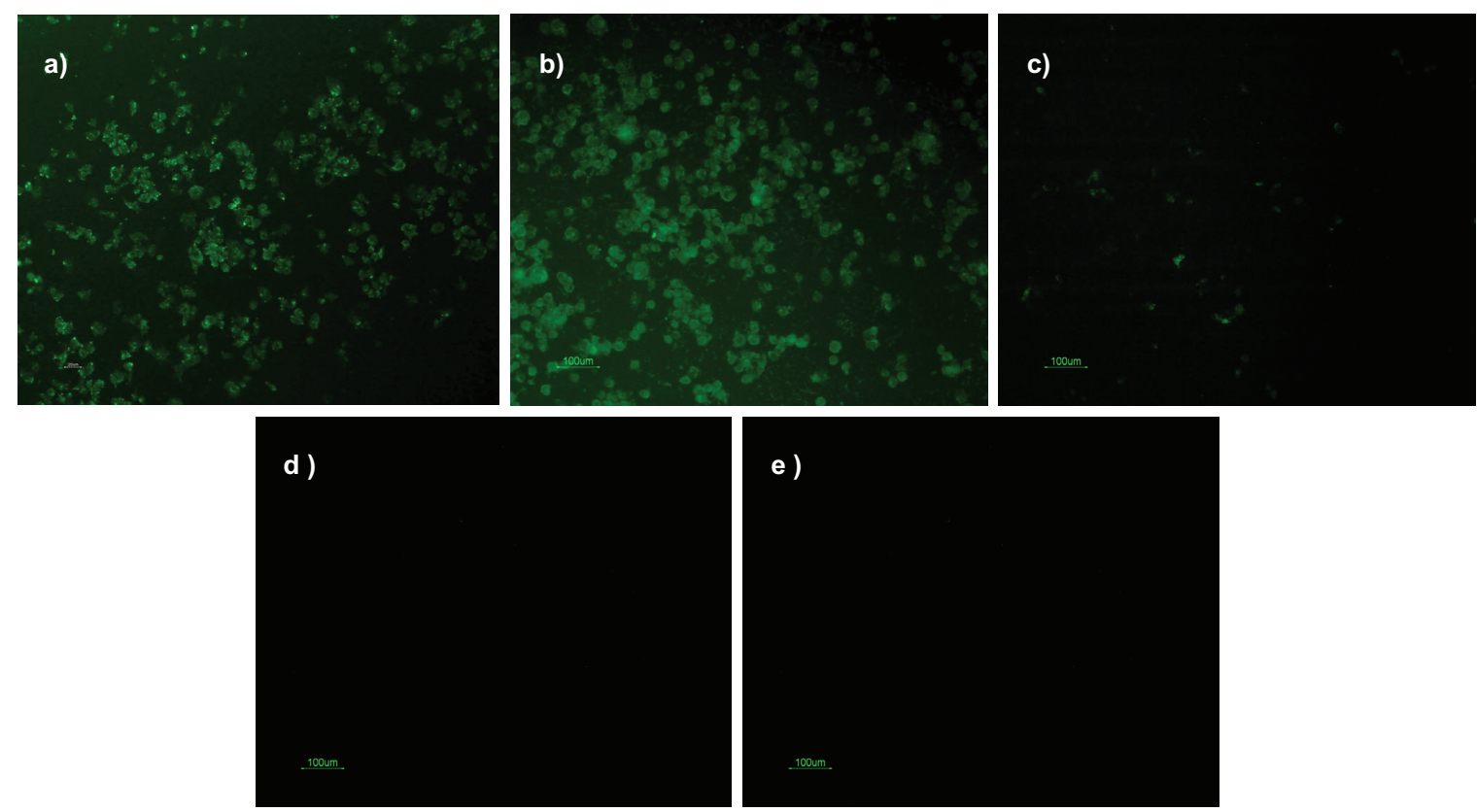

Figure 5 Transfection efficiency of nanoparticles on Neuro2a cells after 4 hours of incubation at $37^{\circ} \mathrm{C}$ and $5 \% \mathrm{CO}_{2}$ : a) PEGylated-chitosan-TPP:siGLO nanoparticles; b) chitosan-TPP:siGLO nanoparticles; c) siGLO only (negative control); d) chitosan-TPP nanoparticles (negative control); e) cells only (negative control)

Abbreviation: TPP, sodium tripolyphosphate.

(95:5 v/v) as a solvent. ${ }^{21}$ However, it was observed that the product obtained did not solubilize homogeneously in most of the organic solvents, which in turn was a hindrance to perform further chemical reactions. Thus, in this chemical scheme we did not incorporate the use of DMF/water (95:5 $\mathrm{v} / \mathrm{v}$ ) as a solvent to synthesize phthaloylated chitosan.

${ }^{13} \mathrm{C}$ NMR data confirm the successful N-phthaloylation of the product. Product (c) in Figure 1 shows the schematic representation of the N-phthaloyl chitosan. The total suppression of side bands (TOSS mode) exhibits peaks at $124.62 \mathrm{ppm}, 131.70 \mathrm{ppm}, 134.42 \mathrm{ppm}$ corresponding to phenylene, and $169.66 \mathrm{ppm}$ and carbonyl group of phthaloyl group and the TOSS - dipolar dephasing (TOSDL) mode confirms the absence of $\mathrm{CH}$ and $\mathrm{CH}_{2}$ peaks due to their short relaxation time and presence of only 2 peaks $131.63 \mathrm{ppm}$

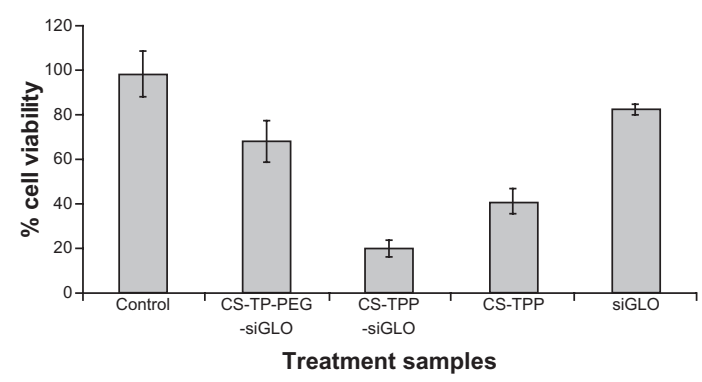

Figure 6 Designed CS-TPP-PEG-siGLO nanoparticle cytotoxicity was investigated using Neuro2a cells. For the experiment, various control nanoparticle formulations were used, the Neuro2a cells were exposed for 4 hours and cell viability was evaluated using spectrophotometer at $490 \mathrm{~nm}$ using standard MTS assays $(n=3)$. and 169.89 ppm assigned to C 1, 2 and carbonyl of phthaloyl group, respectively. The IR data show distinct sharp peaks at $1774 \mathrm{~cm}^{-1}$ and $1702 \mathrm{~cm}^{-1}$ corresponding to imide of phthaloyl group (Figure 2b). The phthaloyl chitosan prepared by this method becomes gel-like when precipitated in water, which supports the data shown by Kurita et al of formation of a uniform structure of phthaloylated chitosan. ${ }^{21}$

The 2-N-phthaloylated chitosan formed was further reacted with thionyl chloride to obtain chlorinated chitosan as represented by product (d) in Figure 1. The IR data in Figure $2 \mathrm{~b}$ show the disappearance of the $\mathrm{OH}$ peak in the range of $3200 \mathrm{~cm}^{-1}$ to $3400 \mathrm{~cm}^{-1}$ in chlorinated phthaloyl chitosan, confirming the replacement of hydroxyl groups with chlorine and the peaks pertaining to imide bond of phthaloyl group $\left(1774 \mathrm{~cm}^{-1}\right.$ and $\left.1710 \mathrm{~cm}^{-1}\right)$ remain intact.

Simultaneously, mPEG $\left(\mathrm{OH}-\mathrm{PEG}-\mathrm{OCH}_{3}\right)$ was activated by $\mathrm{NaH}$ in THF to form PEG alkoxide (-O-PEG-OCH $)_{3}$. To this intermediate, chlorinated phthaloyl chitosan was added to form PEG-grafted phthaloyl chitosan. The desired product was precipitated in methanol, with unreacted PEG being soluble in this solvent. This method is the most direct and the easiest way to obtain PEG-grafted chitosan and does not require any intensive purification procedures. Product (e) in Figure 1 represents the schematic of PEGylated phthaloyl chitosan.

The deprotection of the chitosan was successfully achieved by treating the PEGylated phthaloyl chitosan, product (e) in Figure 1, with hydrazine monohydrate, which 
causes destabilization of the phthaloyl moiety by creating an excess alkaline condition of $\mathrm{pH}>12$. Product ( $\mathrm{f}$ ) in Figure 1 represents the final product achieved: $\mathrm{PEG}$-grafted chitosan. The absence of peaks at $1774 \mathrm{~cm}^{-1}$ and $1710 \mathrm{~cm}^{-1}$ in product (e) confirms the complete dissociation of phthalimido group from chitosan and the appearance of peaks $1633 \mathrm{~cm}^{-1}$ (amide I) and $1582 \mathrm{~cm}^{-1}$ (amide II) corresponds to the presence of primary amine groups in chitosan. The peak at $2871 \mathrm{~cm}^{-1}$ refers to the presence of PEG in product (e), Figure 1. The data on ${ }^{1} \mathrm{H}$ NMR are not shown since it was observed that the multiple peaks of oxymethyl groups in PEG obtained at $\delta 3.3$ to 3.7 cover over the signals of the pyranose ring of chitosan.

The reaction conditions used in the scheme for O-PEGylating chitosan are less intensive in terms of operation and purification. However, $\mathrm{NaH}$, used as a catalyst, makes the reaction conditions alkaline which can potentially have a deleterious effect on the phthaloyl group of chitosan. But, a correct proportion of $\mathrm{NaH}, \mathrm{PEG}$, and chitosan can optimize the reaction conditions. In our study we used 2:4:1 molar ratio of $\mathrm{NaH}: \mathrm{PEG}$ :chitosan. The studies conducted by Makuska's group used $\mathrm{Ag}_{2} \mathrm{O}$ as a catalyst, producing less alkalinity in the solution, suitable for the etherification reaction between chitosan and PEG. The reaction yielded a high degree of substitution of PEG on chitosan, but as stated by the researchers, the residual silver salts that remain in the reaction lead to degradation of chitosan polymer while undergoing deprotection with hydrazine. ${ }^{24}$ Also, the overall yield of the PEGylated chitosan was less and involved 28 to 30 hours of reaction time. Even after implementing several cycles of purification on PEGylated chitosan intermediate, it could not completely remove the silver particles. Thus, the procedure involves intensive and multiple steps to obtain the desired product. The use of $\mathrm{NaH}$ as a catalyst is more promising than using $\mathrm{Ag}_{2} \mathrm{O}$ as a catalyst. In our case, the degree of substitution of PEG on chitosan can be increased by using low molecular weight PEG, but not $<1000$ due to the high solubility of PEG in aqueous as well as organic solvents. We also tried PEGylating chitosan through a scheme followed by Jian and You-Lo, in which phthaloylated chitosan was first swelled in pyridine overnight. ${ }^{25}$ Other researchers have used $\mathrm{NaOH}$ to swell chitosan prior to the reaction in order to achieve homogeneous conditions. $\mathrm{NaOH}$ can deprotect the phthaloyl group on chitosan, and therefore its use was avoided and using pyridine as a solvent was favored due to the solubility of phthaloylated chitosan in pyridine. We obtained similar results in terms of yield of PEGylated chitosan by following the protocol of Jian and You-Lo with the exception of the use of PEG acyl chloride followed by multiple steps that take more time (36-40 hours) in terms of reaction and purification at each step to obtain the final product. The total reaction time employed in this scheme, using $\mathrm{NaH}$, was 18 to 20 hours.

The proposed scheme yielded a moderate $(15 \%-50 \%)$ degree of substitution of PEG on chitosan, which was soluble in acetone, toluene, chloroform, DMF, and ethyl acetate at $50^{\circ} \mathrm{C}$. Also, as reported earlier, O-PEGylated chitosans with free amino groups have a wider application in biotechnology and biomedical systems because of the unchanged poly (glucosamine) skeleton. ${ }^{14}$ As PEG separates the chitosan backbone, thereby decreasing intermolecular hydrogen bonding and making it water soluble, it acts as an important intermediate to carry out further chemical modifications.

Chitosan nanoparticles were prepared by ionic gelation that involves a mixture of 2 aqueous phases, of which one is the polymer chitosan and the other is a polyanion TPP. The positively charged amino group of chitosan interacts with negatively charged TPP to form complex coacervates with a size of about $100 \mathrm{~nm} .{ }^{26-27}$ The nanoparticles formed can have a significant application as a drug/gene carrier system for biomedical applications both in vitro and in vivo. In this study, we have complexed siGLO, a scrambled siRNA with a fluorescent tag with the nanoparticles prepared from the proposed formulation of PEGylated chitosan polymer. Complete complexation of the siGLO was achieved as confirmed by the gel retardation study. The optimal concentration of siGLO to be used for complexation was initially determined by our group and has been published previously as a characterization study. ${ }^{19}$ The PEGylated chitosan polymer complexing siGLO was used as a nanocarrier to transfect a neuronal cell line in order to determine the transfection efficiency of the nanoparticle/carrier. It has been reported by many researchers that PEGylation reduces the transfection efficiency of the nanoparticles since PEG, being neutral in charge, does not efficiently interact with the negatively charged cell membrane. However, in this study, the facile chemoselective substitution of PEG on the C6 position was favored, especially so as not to affect the primary amine groups on chitosan that play a major role is gene complexation and cell transfection.

The transfection study, as shown in Figure 5, confirmed the efficient uptake of PEGylated chitosan nanoparticles (a) compared with chitosan nanoparticles alone (b). This proves that conjugating PEG on the $\mathrm{C} 6$ position of the chitosan polymer did not hinder the inherent character of the chitosan 
polymer to complex and to deliver the siGLO to neuronal cells. To further investigate the effect of nanoparticles on the cells, a cell viability assay was performed. This confirmed the stressed conditions of cells transfected with chitosan nanoparticles alone. The results indicated that although primary amine groups contribute significantly to transfection they may also disrupt the cellular integrity of the cell membrane due to an excessive positive charge on the nanoparticles. The presence of PEG on the nanoparticles reduces steric hindrance and makes nanoparticles more hydrophilic. Also, as observed in Figure 6, the cytotoxicity was greater for the chitosan nanoparticles complexing siGLO than the chitosan nanparticles alone. This could be inferred from a reduction in size of the nanoparticles after complexing siGLO, which in turn allows more particle interaction with the cell membrane, thereby increasing the surface area. The optimization study based on the particle size and zeta potential of chitosan nanoparticles with and without siGLO has previously been published by our group. ${ }^{19}$

\section{Conclusion}

We achieved successful grafting of PEG on chitosan polymer through a novel scheme of forming PEG alkoxide using $\mathrm{NaH}$ as a catalyst. The PEGylated chitosan polymer obtained was easily soluble in water and other organic solvents. The overall procedure is easier and requires less intensive reaction conditions and purification steps to obtain the desired product in a short period of time than the other methods used by other protocols reported in the literature. Also, the procedure does not impart any deleterious effect on the chitosan chain. The PEGylated chitosan nanoparticles successfully complexed siRNA and transfected into the neuronal cell line with minimal cytotoxicity effects. The proposed formulation could have a major implication for biomedical drug targeting strategies involving nanoparticle-mediated targeted delivery.

\section{Acknowledgments}

The authors would like to acknowledge the Canadian Institutes of Health Research (CIHR) grant (MOP-93641) to Dr S Prakash, the support of McGill Major Scholarship to M Malhotra, the support of Industrial Innovation Scholarship (IIS) BMP Innovation - NSERC, FQRNT scholarship and Micropharma Limited Scholarship to Catherine TomaroDuchesneau. We would like to acknowledge the support of the Centre of Biorecognition and Biosensors, the NMR Facility at Quebec/Eastern Canada High Field NMR Center, the Centre for self-assembled Chemical Structures (CSACS) and the facility of Electron Microscopy Research at McGill University. We would like to acknowledge the guidance of Dr Hani Al-Salami for statistical analysis.

\section{Disclosure}

The authors declare no conflicts of interest.

\section{References}

1. Li Q, Dunn ET, Grandmasion EW, Goosen MFA. Applications and properties of chitosan. In: Goosen MFA, editor. Applications of Chitin and Chitosan. Lancaster: Technomic Publishing Company, Inc.; 1992: $3-29$.

2. Ravi-Kumar MNV. A review of chitin and chitosan applications. React Funct Polym. 2000;46:1-27.

3. Bernkop-Schnurch A. Chitosan and its derivatives: potential excipients for peroral peptide delivery systems. Int J Pharm. 2000;194:1-13.

4. Illum L, Jabbal-Gill I, Hinchcliffe M, Fisher AN, Davis SS. Chitosan as a novel nasal delivery system for vaccines. Adv Drug Del Rev. 2001; 51:81-96.

5. Liu WG, Yao KD. Chitosan and its derivatives - a promising non-viral vector for gene transfection. J Control Release. 2002;83:1-11.

6. Sundararajan VM, Howard WTM. Porous chitosan scaffolds for tissue engineering. Biomaterials. 1999;20:1133-1142.

7. Boussif O, Lezoualch F, Zanta MA, Mergny MD, Scherman D, et al. A versatile vector for gene and oligonucleotide transfer into cells in culture and in-vivo - polyethylenimine. Proc Natl Acad Sci U S A. 1995;92:7297-7301.

8. Cho YW, Kim JD, Park K. Polycation gene delivery systems: escape from endosomes to cytosol. J Pharm Pharmacol. 2003;55:721-734.

9. Ishii T, Okahata Y, Sato T. Mechanism of cell transfection with plasmid/ chitosan complexes. Biochim Biophys Acta. 2001;1514:51-64.

10. Skryabin KG, Vikhoreva GA, Varlamov VP. Chitin and chitosan. Production properties and usage. Moscow: Nauka; 2002 [in Russian].

11. Ravi-Kumar MNV, Muzzarelli RAA, Muzzarelli C, Sashiwa H, Domb AJ. Chitosan chemistry and pharmaceutical perspectives. Chem Rev. 2004;104:6017-6084.

12. Sugimoto M, Morimoto M, Sashiwa H, Saimoto H, Shigemasa Y. Chitin and chitosan derivatives. Carbohydr Polym. 1998;36:49-59.

13. Harris JM, Zalipsky S. Poly (ethylene glycol) Chemistry: and Biological Applications. Washington, DC: American Chemical Society: 1997;489.

14. Gorochovceva N, Makuska R. Synthesis and study of water-soluble chitosan-O-poly (ethylene glycol) graft copolymers. European Polymer Journal. 2004;40:685-691.

15. Lin WJ, Lee HG. Design of a microporous controlled delivery system for theophylline tablets. J Control Release. 2003;89:179-187.

16. Lin WJ, Lee HG, Wang DM. The influence of plasticizers on the release of theophylline from microporous-controlled tablets. J Control Release. 2004;99:415-421.

17. Liu X, Howard KA, Dong M, Anderson MO, Rahbek UL, et al. The influence of polymeric properties on chitosan/siRNA nanoparticle formulation and gene silencing. Biomaterials. 2007;28:1280-1288.

18. Nishimura S, Kohgo O, Kuzuhara H, Kurita K. Chemospecific manipulations of a rigid polysaccharide: syntheses of novel chitosan derivatives with excellent solubility in common organic solvents by regioselective chemical modifications. Macromolecules. 1991;24: 4745-4748.

19. Malhotra M, Kulamarva A, Sebak S, Paul A, Bhathena J, et al. Ultrafine chitosan nanoparticles as an efficient nucleic acid delivery system targeting neuronal cells. Drug Dev Ind Pharm. 2009;35: 719-726.

20. Torii Y, Ikeda H, Shimojoh M, Kurita K. Chemoselective protection of chitosan by dichlorophthaloylation: preparation of a key intermediate for chemical modifications. Polym Bull. 2009;62:749-759. 
21. Kurita K, Ikeda H, Yoshida Y, Shimojoh M, Harata M. Chemoselective protection of amino groups of chitosan by controlled phthaloylation: Facile preparation of a precursor useful for chemical modifications. Biomacromolecules. 2002;3:1-4.

22. Calvo P, Remunan-lopez C, Vila-Jato JL, Alonso MJ. Novel hydrophilic chitosan-polyethylene oxide nanoparticles as protein carriers. $J$ Appl Polym Sci. 2007;63:125-132.

23. Kasaai MR. Various methods for determination of the degree of $\mathrm{N}$-acetylation of chitin and chitosan: a review. J Agric Food Chem. 2009;57:1667-1676.

24. Makuska R, Gorochvceva N. Regioselective grafting of poly(ethylene glycol) onto chitosan through C-6 position of glucosamine units. Carbohydr Polym. 2006;64:319-327.
25. Jian D, You-Lo H. PEGylation of chitosan for improved solubility and fiber formation via electrospinning. Cellulose. 2007;14:543-552.

26. Janes KA, Fresneau MP, Marazuela A, Fabra A, Alonso MJ. Polysaccharide colloidal particles as delivery systems for macromolecules. J Control Release. 2001;73:255-267.

27. Pan Y, Li Y, Zhao H, Zheng J, Xu H, et al. Bioadhesive polysaccharide in protein delivery system: chitosan nanoparticles improve the intestinal absorption of insulin in vivo. Int J Pharm. 2002;249:139-147.

\section{Publish your work in this journal}

The International Journal of Nanomedicine is an international, peerreviewed journal focusing on the application of nanotechnology in diagnostics, therapeutics, and drug delivery systems throughout the biomedical field. This journal is indexed on PubMed Central, MedLine, CAS, SciSearch ${ }^{\circledR}$, Current Contents ${ }^{\circledR} /$ Clinical Medicine,
Journal Citation Reports/Science Edition, EMBase, Scopus and the Elsevier Bibliographic databases. The manuscript management system is completely online and includes a very quick and fair peer-review system, which is all easy to use. Visit http://www.dovepress.com/ testimonials.php to read real quotes from published authors. 